\title{
Present bias and the inefficiency of the centralized economy. The role of the elasticity of intertemporal substitution*
}

\author{
Francisco Cabo $^{a}$, Guiomar Martín-Herrán ${ }^{a \dagger}$, María Pilar Martínez-García ${ }^{b}$ \\ ${ }^{a}$ IMUVa, Universidad de Valladolid, Spain $\quad{ }^{b}$ Universidad de Murcia, Spain
}

June 8, 2020

\begin{abstract}
We analyze an endogenous growth model considering agents with an isoelastic utility. Preferences are characterized by a utility affected by a negative externality, and a level of impatience which decays with the time distance from the present. Agents who cannot commit the actions of their future selves, play a game against them. The stationary equilibrium of this game defines a balanced growth path with a slower growth when played by subsequent central planners than when played by decision makers in the market economy. First, we prove that the fast growing market economy implies higher welfare if the negative externality is small, while the centralized economy is welfare improving above a given threshold for the externality (obtained for a specific family of non-constant discount functions). Secondly, we observe that this threshold increases with the elasticity of intertemporal substitution in consumption. Therefore, the greater this elasticity the more likely it is that the externality lies below this threshold, where policy interventions would not
\end{abstract}

*The authors thank Eustasio del Barrio for his invaluable collaboration to prove the existence of a BGP. This study was funded by the Spanish Government (research project ECO2017-82227-P) and by the Regional Government of Castilla y León (research projects VA024P17 and VA105G18), co-financed by FEDER funds.

†Corresponding author: Guiomar Martín-Herrán, Dept. Economía Aplicada (Matemáticas). Facultad de Ciencias Económicas y Empresariales, Universidad de Valladolid, Avda. Valle Esgueva, 6, 47011 Valladolid, Spain. E-mail: guiomar@eco.uva.es 
be adequate. Finally, as one would expect, the range of values of the externality for which the market equilibrium provides higher welfare widens the more different from constant discounting time preferences are, due either to a wider range of variation for the instantaneous discount rates or because these decay more slowly. JEL Classification: D91, O44, C61.

Keywords: Non-constant discounting, elasticity of intertemporal substitution, endogenous growth, Social welfare, time-consistent solutions.

\section{Introduction}

In this paper we consider non-constant discounting characterized by individuals whose level of impatience decreases with the time distance from the present, implying that the future individuals will be more impatient than anticipated today. Thus, as already explained by Strotz (1956) and Pollak (1968), non-constant discounting generates a problem of time inconsistency. An agent who anticipates this problem, and who cannot impose his current decisions on future selves, will play a game against them with the intention of manipulating their behavior. Paradoxically, when this game against future selves is played by a central planner it can lead to a lower social welfare than when played by consumers in a decentralized economy.

This result was highlighted by Krusell et al. (2002) for a Neoclassical growth model, where a central planner is less inclined to invest than agents in a market economy. This occurs because the central planner anticipates that current investment reduces the rate of return of future central planners (and hence their incentive to invest), while current individuals in a decentralized economy take the future rate of return as constant. Likewise, this result is also observed for an endogenous growth model in Cabo et al. (2017), based on the existence of environmental externalities. In this setting, if the externality on utility is large with respect to the externality on production, the market economy grows faster than the centralized economy, where this externality is internalized. Under non-constant discounting, individuals strongly discount the near future, and impatience decreases with the time distance from the present. Thus, when the experiment is controlled to ensure identical overall impatience, these individuals give a relatively stronger value to the long run than individuals with constant discounting. For these long-run

oriented individuals, the faster growth associated with the market economy facilitates greater social welfare. The result is proved for a log-utility. 
The main objective of the paper is to analyze whether the paradoxical result commented above for the endogenous growth model with environmental externalities is still valid if we deviate from the log-utility specification. This specification has been proven a singular case in the literature on economic growth and non-constant discounting (see, Farzin and Wendner 2014 and Cabo et al. 2015). Moreover, we analyze whether this result is more likely to arise when the elasticity of intertemporal substitution in consumption (EIS) is greater or when it is lower than one. With this aim, we define the simplest possible AK growth model with a pollution externality. Production is linear in capital, which generates pollution. The pollution externality is incorporated only in utility, which is non-additive in consumption and pollution. ${ }^{1}$ Non-consumed output accumulates overtime as capital stock. Time-consistent (sophisticated) individuals are aware that future decision makers will deviate from currently-optimal planned consumption and, hence, they play a game against their infinitely many future selves. An equilibrium of this game is a strategy which is optimal for the current self, restricted to the assumption that it will also be played by one's future selves. Focusing on balanced growth paths, ${ }^{2}$ we restrict the analysis to strategies that are linear in the capital stock. Since consumption strategies, output and the dynamics are all linear in the capital stock, the stationary Markov-perfect equilibrium of this inter-generational game constitutes the balanced growth path of the economy. ${ }^{3}$ However, such an equilibrium differs when the game is played by agents in a market economy and when it is played by a sequence of central planners who internalize the environmental externality on utility.

For an EIS different from one, we characterize the existence and uniqueness of a balanced growth path equilibrium in both the centralized and the market economy. Our analysis corroborates, for an EIS different from one, the result in Cabo et al. (2017) that non-constant discounting can lead to lower welfare in the centralized than in the market economy. This result is proven when the environmental externality is small. Moreover, for a family of non-constant discount functions, suggested by Tsoukis et al. (2017), we show that this result is reversed when the environmental externality surpasses a certain threshold. Thus, in contrast with Cabo et al. (2017), we have been able to

\footnotetext{
${ }^{1}$ While for ease of presentation we focus on pollution, one can also think of other negative externalities on utility generated as a by-product from output growth, like congestion problems or an increment in social problems associated with urban growth.

${ }^{2}$ The paper focuses on balanced growth path analysis and as such it omits growth and welfare analysis out of the balanced growth path. Later on it will be proved that no transitional dynamics exists.

${ }^{3} \mathrm{~A}$ description on how to solve this type of games can be found, for example, in Karp 2007, MarínSolano and Navas (2009) or Cabo et al. (2017).
} 
establish that the market economy can lead to higher social welfare for only a moderate environmental externality, i.e. if the environmental concern of individuals is not too large and if production is not excessively pollution-intensive. Interestingly, the interval for the environmental externality within which the market economy provides higher welfare than the centralized solution widens with the EIS. Likewise, this interval is also wider the more different from exponential discounting time preferences are.

A value of the EIS equal to or different from one has been reported to be of great importance in the literature on economic growth and non-constant discounting. Under log-utility, exponential and non-constant discounting are observationally equivalent for Neoclassical growth, as stated in Barro (1999) (or strongly observationally equivalent for endogenous growth in Strulik 2015). Conversely, the observational equivalence is refuted if an isoleatic utility with an EIS different from one is considered (see, Farzin and Wendner 2014 and Cabo et al. 2015, 2016).

Our research question can be framed within the current debate on the actual value of the EIS. The log-utility is a frequent simplifying assumption in theoretical models of economic growth, specifically when they present complexities like non-constant time preferences. However, there is no consensus in the literature about its actual value. As stated by in Thimme (2017), the standard assumption in macroeconomic models of an EIS between 0.5 and 1 (or even lower as in Hall 1988), is being called into question by recent literature, which points to values above 1 (see, for example, Havranek et al. 2015 or Gruber 2013). Moreover, some authors claim that the actual level of EIS can be country specific, and positively correlated with wealth (Havranek et al. 2015, Ben-Gad 2012), or with education, as in Thimme (2017).

To understand the reasons which facilitate a higher social welfare in the market economy under non-constant discounting, and how this result is affected by the pollution externality and by the EIS, it is important to clarify the inter-generational game played by subsequent decision makers when commitment is not feasible. The current central planner (if he could commit his future behavior) would like a large propensity to consume at the present (when he is highly impatient) and a much lower one in the future (more patient). However, knowing that his future self will not be willing to invest that much, he must make a sacrifice and reduce his current consumption. At the equilibrium, his propensity to consume equates his future selves, who will face identical dilemma. The stationary equilibrium to this game can be regarded as a second-best for the central planner. How much the current self is willing to sacrifice present consumption depends 
positively on the EIS. The greater the substitution effect with respect to the income effect, the more strongly current savings raise future utility, inducing a lower propensity to consume at the equilibrium of the game (faster growth). Similar reasoning applies for agents in a decentralized economy with the only difference that they do not internalize the environmental externality, and hence grow faster.

Slower growth in the centralized economy has two welfare implications in this economy. First, a welfare improving effect comes from internalizing the environmental externality. Second, a welfare decreasing effect is associated with slower growth when agents impatience decreases with the time distance from the present. To understand this second effect, note that under non-constant discounting the current agent would like his long-run future selves to consume less than in the equilibrium of the inter-generational game. Because the propensity to consume is lower in the market economy (since the pollution externality is not internalized), this economy can provide higher welfare, at least in the long run. This possibly higher long-run welfare is strongly valued by long-run oriented agents (who strongly discount the near future but less so the distant future). If a first-best equilibrium with commitment were possible, the first effect would dominate. However, this is not necessarily the case when we compare second-best solutions. When there is not commitment and the pollution externality is small, we prove that the second negative effect is stronger and the centralized economy grows too slowly, so the market equilibrium provides a higher social welfare. Moreover, a greater externality would enlarge the positive first effect, which would eventually overcome the negative second effect. In consequence, for a sufficiently large environmental externality, the standard result that social welfare is greater in the centralized solution is found.

Interestingly, we observe that the greater the EIS, the stronger the second negative effect is and, hence, the wider is the range of values for the pollution externality within which the market economy provides higher welfare than the centralized economy. This is because a higher EIS enlarges the weight given to future selves far ahead from the present. Likewise, we also observe that this range of values is also wider the more distant from constant discounting individuals' time preferences are.

These results have a direct policy implication. No intervention is required unless the pollution externality surpasses a threshold. If production is not pollution intensive or pollution has little impact on utility, policy actions aiming to move the market toward the central planner equilibrium are welfare reducing. Moreover, the minimum size of the pollution externality above which policy actions might be adequate is greater the higher 
the EIS is or the more distant individuals are from exponential discounting.

The paper is organized as follows. The next section briefly presents the model for the centralized and the market economies and proves the existence and uniqueness of a balanced path equilibrium. Section 3 compares social welfare under both scenarios and illustrates how the possibility of a market equilibrium which provides higher welfare is affected by changes in the EIS, the pollution externality and the parameters describing the discount function. The conclusions are summarized in Section 4. All proofs are found in the Appendix.

\section{Endogenous growth with a pollution externality}

This section presents an endogenous growth model where an economy produces a final output using linear technology, and generates pollution as a by-product:

$$
Y(K)=A K, \quad P(K)=K^{\lambda} \text { with } \lambda>0 .
$$

Capital, $K$, is the only input, and $P$ represents pollution. Assuming constant population normalized to one, here and henceforth all variables are in per capita terms. Pollution negatively enters the utility function, which also depends positively on consumption, $C$ :

$$
U(C, P)= \begin{cases}\frac{\left(C P^{-\phi}\right)^{1-1 / \sigma}-1}{1-1 / \sigma} & \text { if } \sigma \neq 1 \\ \ln C-\phi \ln P & \text { if } \sigma=1 .\end{cases}
$$

Utility is assumed to be isoelastic with a constant elasticity of intertemporal substitution in consumption, $\sigma \in(0, \infty)$. Parameter $\phi>0$ measures how strongly pollution affects utility. Moreover, taking into account the pollution elasticity of capital in (1), the utility can be written in terms of consumption and capital, with the term $\phi \lambda$, henceforth denoted $\varphi$, defining the pollution externality in this economy. Hereinafter we will assume that this externality satisfies the condition $^{4} \varphi<1$. Under this condition, along a balanced path, the positive effect of a constant growth in consumption on utility is stronger than the negative effect of a sustained growth in pollution (associated with the growth in the capital stock).

\footnotetext{
${ }^{4}$ Along the balanced path, where consumption, capital and output grow at the same constant rate, this condition guarantees that the maximized Hamiltonian satisfies the Arrow sufficient optimality conditions.
} 
The cross-effect of consumption and pollution on utility crucially depends on the EIS. When $\sigma<1$, there exists a "compensation effect" of pollution on consumption, $\partial^{2} U /(\partial c \partial P)>0$. The growth in pollution partially counterbalances the fall in the marginal utility of consumption with increasing levels of consumption. This offsetting effect is weaker the greater the EIS, and actually turns into a fostering effect for $\sigma>1$, when there is a "distaste effect" of pollution on consumption: $\partial^{2} U /(\partial c \partial P)<0$. In the usual assumption of log-utility, utility is separable in consumption and pollution and no distaste or compensation effect exists.

We assume agents with a decreasing level of impatience, who discount the future at a rate which decays with the time distance from the present, $j$. Thus, the instantaneous discount rate $\rho(j)$ satisfies $\rho(j)>0, \dot{\rho}(j)<0, \forall j \geq 0$, with $\lim _{t \rightarrow+\infty} \rho(j)=\rho_{\infty}>0$. In consequence, the discount function $\theta(j)$ must satisfy $\dot{\theta}(j) / \theta(j)=-\rho(j)$ with $\theta(0)=1$. Therefore, this function reads:

$$
\theta(j)=e^{-\int_{0}^{j} \rho(z) d z} .
$$

This economy can be compared to a standard economy with constant discounting under the assumption of identical overall impatience:

$$
\int_{0}^{\infty} e^{-\hat{\rho} j} d j=\int_{0}^{\infty} \theta(j) d j .
$$

where the constant discount rate, $\hat{\rho}$, with an equivalent overall level of impatience to (3), is defined as:

$$
\hat{\rho}=\left[\int_{0}^{\infty} \theta(j) d j\right]^{-1} .
$$

Under the standard assumption of constant discounting, the growth rate along the balanced growth path for an AK-type model with a pollution externality, would read $\sigma[A-\hat{\rho} /(1-\varphi)]$. In this economy, a positive growth requires a not excessively large externality and a sufficiently large marginal productivity of capital with respect to the discount rate (assumed henceforth):

$$
A(1-\varphi)>\hat{\rho} .
$$

In this paper we consider individuals with non-constant preferences, who become more patient as the time distance from the present widens. We consider sophisticated agents who acknowledge that the preferences of their future selves will change, and hence, play a game against all their future selves. This hypothesis guarantees time consistency: the optimal solution computed at the current time also remains valid if recomputed 
by future generations. Time-consistent solutions are characterized for both individual agents in a decentralized economy and for the central authority in a centralized economy, who shares the preferences of the current selves.

In the centralized economy, at current date $t$, the central planner maximizes the lifetime utility of the representative consumer (t-agent). He chooses the instantaneous consumption under the assumption that all his future selves will follow the same linear strategy $C_{t}(\tau)=\xi K_{t}(\tau), \tau \geq t$, and $\xi$ constant. These future selves will solve an identical problem (except for the initial value of the capital stock). The only possible Markov-perfect equilibrium to this sequence of games is the stationary strategy with a constant propensity to consume. This equilibrium characterized a balanced growth path. In this game, the central planner at each time $t$, takes into account that the decision to raise capital also enhances production and, consequently, pollution:

$$
\begin{array}{ll} 
& \max _{C_{t}} \int_{t}^{\infty} U\left(C_{t}(\tau), K_{t}^{\lambda}(\tau)\right) \theta(\tau-t) d \tau, \\
\text { s.t.: } & \dot{K}_{t}(\tau)=A K_{t}(\tau)-C_{t}(\tau), \quad K_{t}(t)=K_{t}, \\
& C_{t}(\tau)=\xi K_{t}(\tau), \quad \tau \geq t,
\end{array}
$$

where the utility function is given by (2), and $\tau-t$ denotes the time distance from the present.

In a market economy, the representative household at time $t$ plays a game with his future selves similar to the game described for the central planner. He maximizes his utility from current time $t$ on, taking pollution as given and subject to the budget constraint:

$$
\begin{array}{ll} 
& \max _{c_{t}} \int_{t}^{\infty} U\left(c_{t}(\tau), P_{t}(\tau)\right) \theta(\tau-t) d \tau, \\
\text { s.t.: } & \dot{k}_{t}(\tau)=r k_{t}(\tau)-c_{t}(\tau), \quad k_{t}(t)=k_{t}, \\
& c_{t}(\tau)=\xi k_{t}(\tau), \quad \tau \geq t,
\end{array}
$$

where $k_{t}(\tau)$ denotes household assets and $r$ the market interest rate. ${ }^{5}$ Unlike the central planner, the households in the market solution disregard how an increment in the capital stock will induce higher future pollution.

Following standard modelization in A $K$-type models, we assume a large number of identical firms that produce following a linear technology, $Y_{i}=A K_{i}$. Considering profit

\footnotetext{
${ }^{5}$ Note that without labor, consumers' wealth is exclusively determined by the capital stock they own and lend to firms.
} 
maximizing firms, the rental price to capital equals the capital productivity, $r=A$. Finally, assuming identical firms of measure one, $K=K_{i}$ and consequently, $P=P_{i}=$ $K^{\lambda}$, and the aggregate production in (1) follows.

Note that in both the central planner and the market economy, the stationary Markov-perfect equilibrium of the inter-generational game constitutes the balanced growth path. This result comes from the assumption of stationary linear strategies for an AKmodel.

In the centralized economy consumption and capital grow, along the balanced growth path, at the same constant rate, $g$, and the propensity to consume out of wealth (capital) remains constant, denoted by $\xi$. Correspondingly, from (1) pollution grows at rate $\lambda g$. Thus, considering an EIS different from one, ${ }^{6}$ the accumulated flow of discounted utility along a balanced path can be written as

$$
\int_{t}^{\infty} U\left(\xi K_{t}(\tau), K_{t}^{\lambda}(\tau)\right) \theta(\tau-t) d \tau=-\frac{\sigma}{(\sigma-1) \hat{\rho}}+\sigma \frac{\xi^{1-\frac{1}{\sigma}}}{\sigma-1} K_{t}^{-\eta} \Omega(g, \sigma, \varphi),
$$

with $K_{t}(\tau)$ the capital stock in the economy along a balanced path and

$$
\Omega(g, \sigma, \varphi)=\int_{0}^{\infty} \theta(j) e^{-\eta g j} d j, \quad \eta=\frac{1-\sigma}{\sigma}(1-\varphi) .
$$

In (14) the time distance from the present is denoted by $j=\tau-t$.

In the market economy along a balanced growth path, household consumption and assets grow at the same rate, $g$, which also determines the growth rate of the total capital in the economy. The propensity to consume out of capital is described as the consumption by each household divided by that household's assets. Thus, the accumulated flow of discounted utility in the market economy reads:

$$
\int_{t}^{\infty} U\left(\xi k_{t}(\tau), K_{t}^{\lambda}(\tau)\right) \theta(\tau-t) d \tau=-\frac{\sigma}{(\sigma-1) \hat{\rho}}+\sigma \frac{\xi^{1-\frac{1}{\sigma}}}{\sigma-1} k_{t}^{-\eta_{1}} K_{t}^{-\eta_{2}} \Omega(g, \sigma, \varphi),
$$

with $K_{t}(\tau)$ and $k_{t}(\tau)$ the capital stock in the economy and the households assets along a balanced path and

$$
\eta_{1}=\frac{1-\sigma}{\sigma}, \quad \eta_{2}=-\frac{1-\sigma}{\sigma} \varphi
$$

Note that $\eta_{1}+\eta_{2}=\eta$. The sign of $\eta$ depends on whether the EIS is greater or lower than one. The assumption of an income effect stronger than or equal to the substitution effect is the most commonly accepted assumption in the literature. Under this assumption $\eta>$ 0 and the improper integral $\Omega(g, \sigma, \varphi)$, which defines discounted utility, is convergent.

\footnotetext{
${ }^{6}$ The analysis of the particular case $\sigma=1$ is postponed until Remark 2 .
} 
In contrast, under the alternative assumption of $\sigma>1, \eta<0$ and one needs to establish a ceiling for the elasticity of intertemporal substitution in consumption to guarantee convergence (condition (19) in the following proposition), as also occurs in economic growth models with constant discounting.

Proposition 1 The growth rate along a balanced path equilibrium is characterized by

$$
\begin{gathered}
g^{S P}=\sigma \frac{(1-\varphi) A-\Delta\left(g^{S P}, \sigma, \varphi\right)}{1-\varphi} \equiv h^{S P}\left(g^{S P}\right) \quad \text { [Social Planner Economy] } \\
g^{D E}=\sigma \frac{A-\Delta\left(g^{D E}, \sigma, \varphi\right)}{1-\varphi+\sigma \varphi} \equiv h^{D E}\left(g^{D E}\right) \quad \text { [Decentralized Economy] }{ }^{7}
\end{gathered}
$$

with

$$
\Delta(g, \sigma, \varphi)=\int_{0}^{\infty} \rho(j) \omega(j) d j, \quad \omega(j)=\frac{\theta(j) e^{-\eta g j}}{\Omega(g, \sigma, \varphi)}
$$

which are feasible under condition

$$
\sigma<\hat{\sigma} \equiv 1+\frac{\rho_{\infty}}{(1-\varphi) A-\rho_{\infty}}
$$

Condition (19) guarantees the convergence of the improper integrals $\Omega(g, \sigma, \varphi)$ and $\Delta(g, \sigma, \varphi)$ with a positive growth rate, $g$. Note that, under condition (6) and taking into account that $\hat{\rho}>\rho_{\infty}$, then $\hat{\sigma}>1$.

The role played by $\Delta(g, \sigma, \varphi)$ in (16) and (17) resembles that of the constant discount rate in the Ramsey rule. Then, following Barro (1999), it is interpreted as the effective rate of time preference. This term depends on the growth rate of the economy, the EIS and the environmental externality.

Remark 2 In the particular case $\sigma=1$, the accumulated flow of discounted utility along a balanced path (13) in the centralized economy, and (15) in the market economy translates into

$$
\begin{aligned}
& \int_{t}^{\infty}\left[\ln \left(\xi K_{t}(\tau)\right)-\phi \ln \left(K_{t}^{\lambda}(\tau)\right)\right] \theta(\tau-t) d \tau=\frac{1}{\hat{\rho}}\left[(1-\varphi) \ln \left(K_{t}\right)+g(1-\varphi) \bar{J}^{1}+\ln \xi\right], \\
& \int_{t}^{\infty}\left[\ln \left(\xi k_{t}(\tau)\right)-\phi \ln \left(K_{t}^{\lambda}(\tau)\right)\right] \theta(\tau-t) d \tau=\frac{1}{\hat{\rho}}\left[\ln \left(k_{t}\right)-\varphi \ln \left(K_{t}\right)+g(1-\varphi) \bar{J}^{1}+\ln \xi\right],
\end{aligned}
$$

where $\bar{J}^{1}=\hat{\rho} \int_{0}^{\infty} j \theta(j) d j$.

Note also that $\Omega(g, 1, \varphi)=1 / \hat{\rho}$ and $\Delta(g, 1, \varphi)=\hat{\rho}$. In consequence, expressions (16)

\footnotetext{
${ }^{7}$ Here, and henceforth, superscript $S P$ refers to the Social Planer economy and $D E$ to the Decentralized economy.
} 
and (17) explicitly define the equilibrium growth rates in the centralized and the market economies:

$$
g^{1 S P}=\frac{(1-\varphi) A-\hat{\rho}}{(1-\varphi)}, \quad g^{1 D E}=A-\hat{\rho}
$$

In the case of $\sigma \neq 1$, the following proposition proves that equations (16) and (17) implicitly define the balanced growth rates in the centrally planned and the decentralized economies. The proposition also proves that the equilibrium growth rate is larger in the market economy. ${ }^{8}$

Proposition 3 Under conditions (6) and (19) there exists a unique balanced path equilibrium, for both the centrally planned and the market economies, with positive growth rates, $g^{S P}$ and $g^{D E}$. These rates are the unique solutions for equations (16) and (17), respectively. Moreover, $g^{D E}>g^{S P}$.

With the aim of providing an intuition on how non-constant discountig affects the long-run growth rate of both economies, we rewrite the first-order optimality conditions in (26) and (32) with the help of expressions (28) and (33) as

$\frac{\partial U\left(C_{t}, K_{t}^{\lambda}\right)}{\partial C_{t}}=\left[(1-\varphi) \Omega\left(g^{\mathrm{SP}}, \sigma, \varphi\right)\right]^{\frac{1}{\sigma}} K_{t}^{-(1+\eta)}, \quad \frac{\partial U\left(c_{t}, K_{t}^{\lambda}\right)}{\partial c_{t}}=\Omega\left(g^{\mathrm{DE}}, \sigma, \varphi\right)^{\frac{1}{\sigma}} k_{t}^{-\left(1+\eta_{1}\right)} K_{t}^{-\eta_{2}}$,

for the centralized and the market solutions, respectively. These equations balance current marginal utility from consumption to the present value of all ongoing effects on utility of a marginal increment in current capital. These ongoing effects depend on the current capital stock, the term $\Omega(g, \sigma, \varphi)$ and, in the centralized economy (where the pollution externality is internalized), on $1-\varphi$. To give an interpretation to expression $\Omega(g, \sigma, \varphi)$, note that from (2) it follows that along a balanced path, the utility from a current time $t$ on evolves according to the term $e^{-\eta g j}$. Therefore, this term determines the marginal effect that changes in current capital will have on the utility $j$ periods from $t$ along a balanced path. If $\sigma<1$, the income effect exceeds the substitution effect, then $\eta>0$ and marginal future effects shrink with the time distance from the present. In contrast, if $\sigma>1$, then $\eta>0$ and current changes induce exploding marginal future effects. Finally, the assumption of log-utility is consistent with an invariant effect of a current change on all future utilities. The effects for different values of $\sigma$ are depicted in Figure 1 (left).

\footnotetext{
${ }^{8}$ This result is not due to non-constant discounting. An identical result is found in Smulders and Gradus (1996) under exponential discounting.
} 

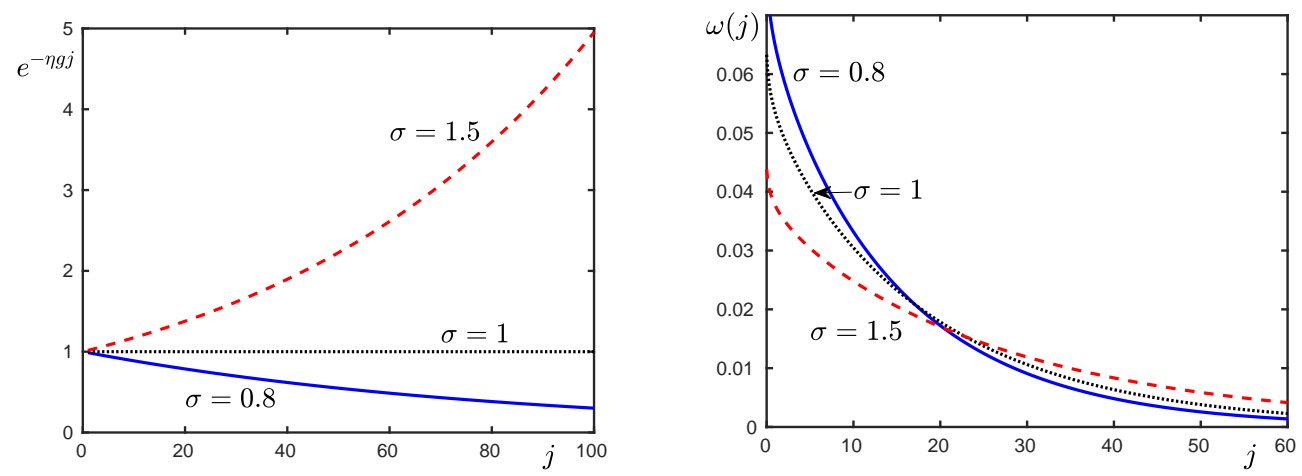

Figure 1: Future marginal effects in utility (left); and weights $\omega(j)$ (right).

A current $t$-agent discounts all these future effects according to the discount function, $\theta(j)$. Therefore, $\Omega(g, \sigma, \varphi)$ collects the total effect of a marginal increment in current capital on all future utility as the aggregated sum of the discounted value of ongoing future marginal effects on utility. This total effect can be decomposed in the effect $j$ periods from now for every $j \geq 0$, which in relative terms is defined by $\omega(j)$ in (18), and depicted in Figure 1 (right). These relative future effects can also be interpreted as the weights defining the effective rate of time preference, $\Delta(g, \sigma, \varphi)$, as a weighted mean of the instantaneous discount rates from the present time on, $\rho(j)$, with weights satisfying $\int_{0}^{\infty} \omega(j) d j=1$. The next proposition describes the relationship between $\Delta(g, \sigma, \varphi)$ and $g$.

Proposition 4 The effective discount rate increases (decreases) with the growth rate of the economy if the EIS is lower (greater) than one, and it is unaffected for a log-utility.

A higher growth rate differently impacts on the effective discount rate depending on whether the EIS is above or below one. For $\sigma<1$ (i.e. $\eta>0$ ) the decay in the marginal effect of current changes on future utility, $e^{-\eta g j}$, is more pronounced the greater the growth rate of the economy. Thus, a higher growth rate raises the weights given to the instantaneous discount rates closer to the present (which take higher values). In consequence, it also raises the effective discount rate (i.e. individuals are more short-run oriented). In contrast, for $\sigma>1$ (i.e. $\eta<0$ ), the greater the growth rate of the economy is, the faster the marginal future effects, $e^{-\eta g j}$, explode. Thus, a higher growth rate raises the weights given to the instantaneous discount rates far ahead in the future (which take lower values). In consequence, it reduces the effective discount rate (i.e. individuals are more long-run oriented). Finally, when the income and the substitution effect exactly 
cancels out (i.e. $\sigma=1$ ), growth has no effect on the distribution of weights. ${ }^{9}$

Our analysis has focused on the balanced path equilibrium. The next proposition shows that, as in the standard AK-model with exponential discounting, no transitional dynamics exists. ${ }^{10}$

Proposition 5 The balanced path equilibrium in the centralized or in the market economy shows no transitional dynamics.

\section{Social welfare comparison}

We are now in the position to answer one of the main research questions of this paper, concerning the social welfare comparison between the centralized and the market economy. Analytical results are presented as propositions. To illustrate these analytical results, and to present additional numerical results, we carry out a numerical analysis for a particular family of discount functions presented in Tsoukis et. al. (2017). These functions can be embodied as

$$
\theta(j)=e^{-\rho_{\infty} j}(1+\delta j)^{-\gamma / \delta}
$$

with $\rho_{\infty}>0, \gamma \in(0,1), \delta>0$ and $\gamma / \delta<1$. In consequence, the instantaneous discount rate associated with this expression is

$$
\rho(j)=\rho_{\infty}+\gamma /(1+\delta j)
$$

Therefore, $\rho(j)$ decreases from $\rho_{\infty}+\gamma$ to $\rho_{\infty}$ as the time distance from the present tends to infinity. In the numerical simulations, we take as benchmark the following parameter values: $\gamma=0.5, \delta=10, \rho_{\infty}=0.05, K_{t}=1, \varphi=0.2$ and $A=0.12$.

In the previous section, considering a pollution externality in the utility of consumption, we saw that the market economy grows faster than the centralized economy. Faster growth might lead to higher welfare in the decentralized scenario when individuals discount the future at a decreasing rate. This result crucially depends on the three main ingredients in this economy: the size of the environmental externality, the EIS and the

\footnotetext{
${ }^{9}$ Alternatively, we could refer to the effect of $g$ on $\Omega(g, \sigma, \varphi)$. A faster growth reduces the total effect of a marginal increment of present capital on ongoing utility if $\sigma<1$, increasing it if $\sigma>1$, or leaving it unchanged if $\sigma=1$.

${ }^{10}$ We thank one anonymous reviewer for bringing our attention to this analysis.
} 
way individuals discount the future. In this section we analyze how these ingredients influence the equilibrium growth rates and the welfare comparison in the two economies.

First, we focus on the pollution externality, $\varphi$, which represents either the impact of pollution on utility, $\phi$, or the elasticity of pollution with respect to capital, $\lambda$. The next proposition describes its effect on the growth rates.

Proposition 6 The equilibrium growth rate in the centralized economy decreases with the pollution externality.

The equilibrium growth rate in the market economy increases (decreases) with the pollution externality if the EIS is lower (greater) than one, and is not influenced by the externality for a log-utility.

A pollution externality has a direct and an indirect effect on growth. The direct effect mimics the standard effect under constant discounting and can be decomposed into two separated effects. First, when the externality is internalized in the centralized economy it discourages savings and growth. Second, if the EIS is different from one, a value of $\sigma$ below one implies a compensation effect which boosts growth, while a value of $\sigma$ above one implies a distaste effect which slows down growth. This second effect applies in both the market and in the centralized economies.

Due to the assumption of non-constant discounting, a greater externality also has an indirect effect on growth, which is collected by its effect on the effective discount rate, $\Delta(g, \sigma, \varphi)$. From (14) it follows that the marginal effect of current changes on future utilities, $e^{-\eta g j}$, shrinks more smoothly (for $\sigma<1$ ), or expands less rapidly (for $\sigma>1$ ). Hence, for $\sigma<1$, individuals become more long-run oriented, their effective discount rate decreases, inducing a rise in the growth rate. The opposite applies for $\sigma>1$.

For the centralized equilibrium the total effect is negative, as shown in the proof of Proposition 6 in the Appendix. For the market equilibrium, a greater externality induces faster growth for a low EIS, and slower growth for a large EIS. This can be observed in Figure 2.

The size of the pollution externality is also crucial when comparing social welfare in the centralized and the market economies. The existence of a pollution externality leads the central planner to slow down growth below the market equilibrium. The lower growth has a twofold effect on welfare: direct positive effect, because the pollution externality is internalized, and a negative effect when impatience decreases with the time distance from the present. Under these time preferences, the current agent would like (if he could 

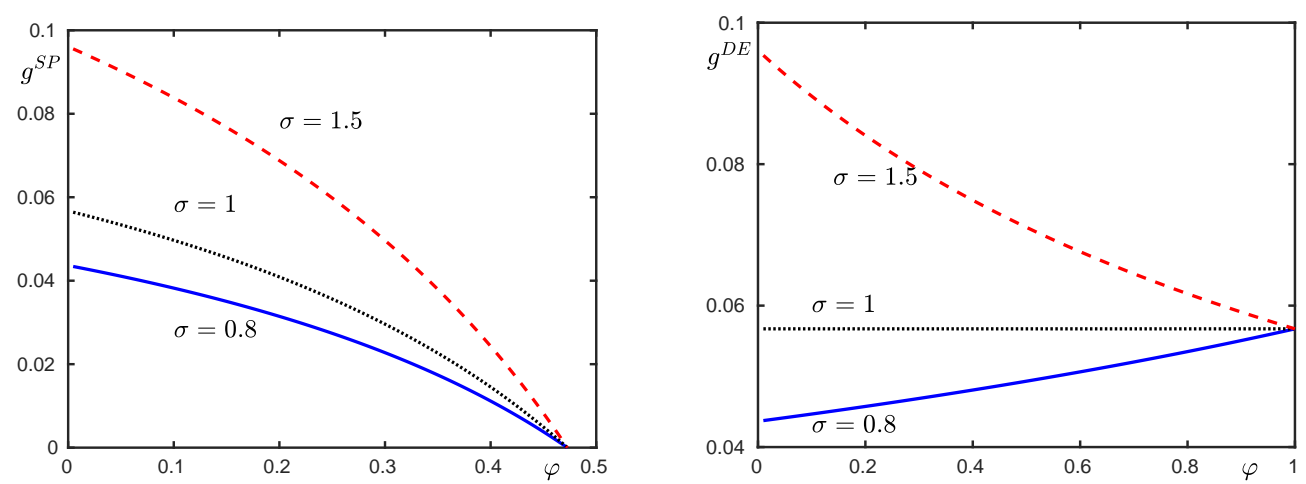

Figure 2: Sensitivity of $g^{\mathrm{SP}}$ (left) and $g^{\mathrm{DE}}$ (right) w.r.t. $\sigma$ and $\varphi$.

commit future actions) a time-decaying propensity to consume. However, without the possibility to commit future actions, the current agent must follow a second-best policy, which stems from the inter-generational game with his future selves. The stationary equilibrium of this game imposes a constant propensity to consume, which from the standpoint of the current agent is too low in the short run and too high in the long run. In consequence, for this agent the smaller propensity to consume in the market economy in the long run can be welfare enhancing. The following proposition proves that the second negative effect can overcome the direct positive effect of the slower growth in the centralized economy.

Proposition 7 Under conditions (6) and (19) the social welfare in the market economy surpasses the social welfare in the centralized economy provided that the pollution externality is sufficiently small.

Proposition 7 clearly states that the direct positive welfare effect of internalizing the pollution externality can be outweighed by the negative welfare effect of a too low growth rate in the long run, from the standpoint of the current agent. This analytical result is valid if the pollution externality is sufficiently small. Next we analyze whether this result can be generalized for any size of this externality or if it is reversed at some threshold. While this question cannot be analytically answered, we rely on a numerical analysis for the discount functions in (21). For these discount functions we can numerically compute the growth rates and the value functions for the centralized and the market economies, in (27)-(30) and (33)-(34) for $\sigma \neq 1$, and in (35)-(36) for $\sigma=1$. Furthermore, we compute the relative gap in social welfare between the market and the centralized equilibria: $\left(W^{\mathrm{DE}}\left(K_{t}, K_{t}\right)-W^{\mathrm{SP}}\left(K_{t}\right)\right) /\left|W^{\mathrm{SP}}\left(K_{t}\right)\right|$. This relative gap is computed for the pollution externality, $\varphi$, running from 0 to 1 . As proved in Proposition 7 , the market 
economy provides higher social welfare than the centralized solution when the pollution externality is small. As this externality becomes bigger, Figure 3 shows that the gap ${ }^{11}$ widens initially but narrows later on, and it eventually turns negative. This analysis highlights that when individuals discount at a decreasing rate, there exists a window for the pollution externality within which the market solution provides higher welfare, but above a given threshold, $\hat{\varphi}$, the standard result of a higher social welfare in the centralized economy arises. This is true regardless of whether we consider an EIS above, below or equal to 1 . According to this result, policy actions might be adequate if the pollution externality exceeds this threshold, but they are not when pollution has a small impact on consumers' utility. We have checked that this result is qualitatively robust to changes in the parameter values.

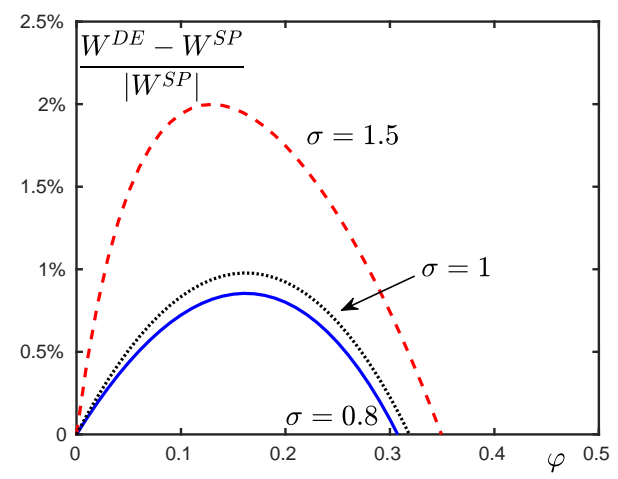

Figure 3: Relative gap in social welfare: centralized vs. market.

The EIS is a second key element in consumption savings decisions, and hence it fundamentally determines the gap in growth rates and social welfares between the centralized and the market economy. As can be observed in Figure 2, the next proposition establishes a total positive effect of the EIS on the growth rate in both economies.

Proposition 8 The equilibrium growth rates in the centralized and the market economy are greater the higher the elasticity of intertemporal substitution in consumption.

The EIS has a twofold effect on growth: A direct positive effect (likewise as under constant discounting) stems from a softer decay in the marginal utility of consumption with higher consumption levels. Along the balanced path pollution also increases, which partially counterbalances the fall in the marginal utility of consumption when $\sigma<1$,

\footnotetext{
${ }^{11}$ The gap is computed in relative terms and we observe that, when positive, it can represent a maximum between $0.7 \%$, for $\sigma=0.8$, and $2 \%$, for $\sigma=1.5$.
} 
and there exists a "compensation effect" of pollution on consumption; or is exacerbated when $\sigma>1$ and a "distaste effect" appears. All in all, a larger EIS has a direct positive effect on growth, which is more intense in the centralized economy, which anticipates the effect of consumption decisions on future pollution. This effect is reinforced by a second effect stemming from the assumption of non-constant discounting. A higher EIS implies a stronger relative effect of current changes on far distant utilities, when the level of impatience is lower. This is equivalent to having a lower effective discount rate which indeed induces an even faster growth.

The numerical analysis shows that a higher EIS monotonously implies a wider relative gap $\left(W^{\mathrm{DE}}\left(K_{t}, K_{t}\right)-W^{\mathrm{SP}}\left(K_{t}\right)\right) /\left|W^{\mathrm{SP}}\left(K_{t}\right)\right|$. This can be observed in Figure 3 for three different values of $\sigma$. This is because a higher EIS enlarges the negative welfare effect that individuals with non-constant time preferences associate to the low growth rate in the centralized economy, when they would prefer a faster growth in the long run (as in the market economy). In consequence a greater pollution externality is required for the positive welfare effect of internalizing the externality to overcome the negative effect just described. Thus, a higher EIS widens the range of values for the pollution externality, $\hat{\varphi}$, within which the market equilibrium provides higher welfare and no policy actions are needed. Increasing $\sigma$ till the upper bound $\hat{\sigma}$ (above which the effective rate of discount, $\Delta(g, \sigma, \varphi)$, is not well defined $), \hat{\varphi}$ monotonously increases, as long as the growth rate of the economy is positive.

Finally, we analyze the impact of non-constant discounting on the growth rates and the welfare in the centralized and the market economies. We aim to characterize how growth is affected by the way individuals discount future utility, but maintaining the overall level of impatience unchanged. The overall level of impatience in (5), considering the Laplace transform, can be stated as

$$
\hat{\rho}=\frac{e^{-\rho_{\infty} / \delta} \delta^{\gamma / \delta} \rho_{\infty}^{1-\gamma / \delta}}{\Gamma\left(1-\frac{\gamma}{\delta}, \frac{\rho_{\infty}}{\delta}\right)},
$$

where $\Gamma(a, b)=\int_{b}^{\infty} x^{a-1} e^{-x} d x$ is the incomplete gamma function. ${ }^{12}$ Thus, we perform a controlled experiment, and analyze the effect of $\gamma$ and $\rho_{\infty}$, when $\delta$ adjusts so that condition (22) remains unaltered.

As shown in Figure 4, an increment in $\gamma$ widens the range for the instantaneous discount rate, $\left[\rho_{\infty}+\gamma, \rho_{\infty}\right)$, while a lower $\rho_{\infty}$ implies a slower decay of the instantaneous

\footnotetext{
${ }^{12}$ This follows the expression proposed by Tsoukis et al. (2017). We have corrected a mistake by changing the standard Gamma function for an incomplete Gamma function.
} 

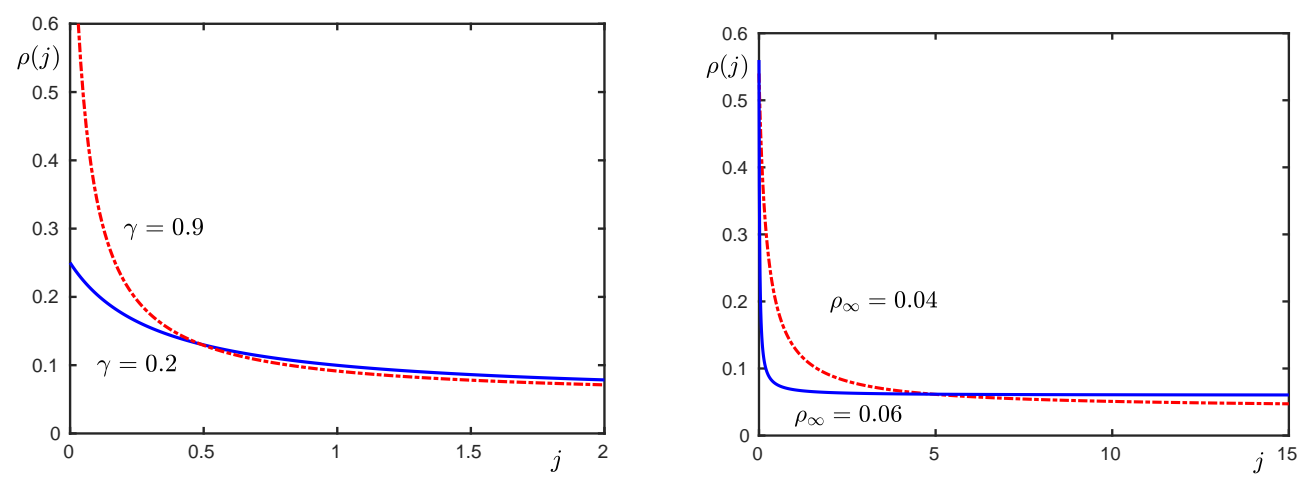

Figure 4: Effect of $\gamma$ (left) and $\rho_{\infty}$ (right) on $\rho(j)$.

discount rate. Thus, the discount function is more distant from constant discounting the higher $\gamma$ or the lower $\rho_{\infty}$. Both changes imply more long-run oriented individuals. Being more long-run oriented has a different effect on growth depending on whether the EIS is above or below 1 .

A more long-run oriented individual would slow down growth if the effect of changes in current capital on future utility decreases with the time distance from the present (an EIS lower than 1). For this agent, the aggregate value of the ongoing effects, $\Omega(g, \sigma, \varphi){ }^{13}$ is reduced, together with the incentive on current savings. By contrast, this agent would speed up growth if, conversely, changes in current capital on future utility expand with time (an EIS greater than 1). Figure 5 depicts the effect of a rise in $\gamma$, from 0.2 to 0.9 . For $\sigma<1$ (left chart) the growth rate is reduced more strongly, the smaller the pollution externality. Conversely, for $\sigma>1$ (right chart) the growth rate is increased, and the gap between the two scenarios widens with the intensity of the externality. Identical reasoning applies for a lower $\rho_{\infty}$.

Finally, we show that the more the agents preferences differ from exponential discounting, the wider is the interval for the pollution externality compatible with a welfare enhancing market equilibrium. This is numerically shown by computing the value of the threshold $\hat{\varphi}$ as an increasing function of $\gamma$, shown in Figure 6. Thus, the wider the range in instantaneous discount rates, the wider the interval of the pollution externality within which policy intervention would not be needed. A similar analysis and result arise for a smaller $\rho_{\infty}$. Note that Figure 6 shows that the threshold also widens with the EIS, as already observed in Figure 3.

\footnotetext{
${ }^{13}$ Note that a reduction in $\Omega(g, \sigma, \varphi)$ is equivalent to an increment in the effective discount rate, from equation (30), and the consequent decay in the growth rate of the economy.
} 

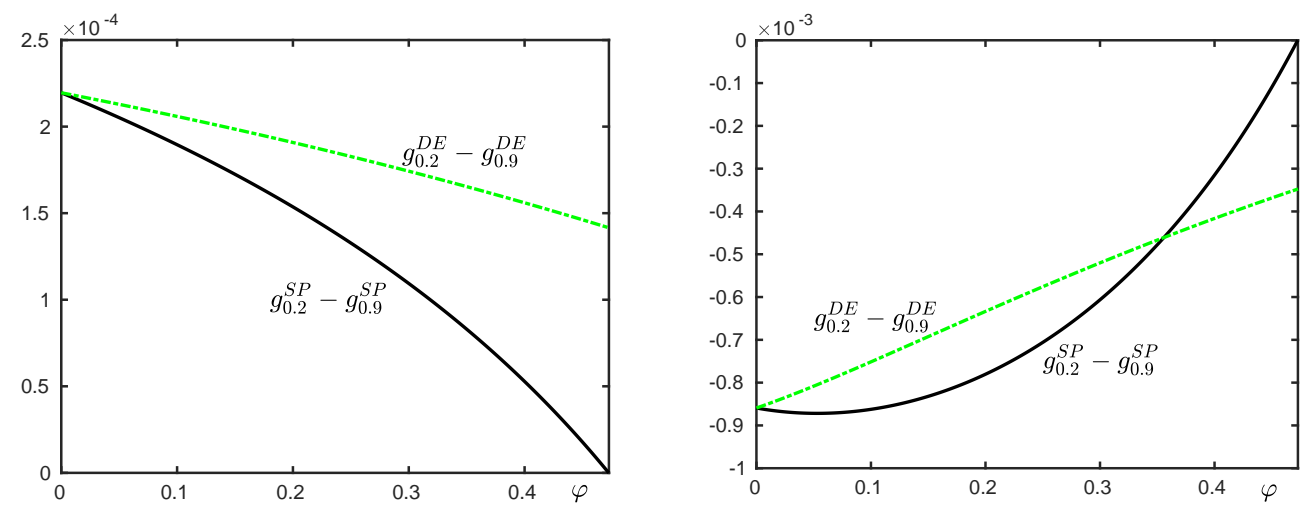

Figure 5: Effect of $\gamma \cdot \sigma=0.8$ (left); $\sigma=1.5$ (right).

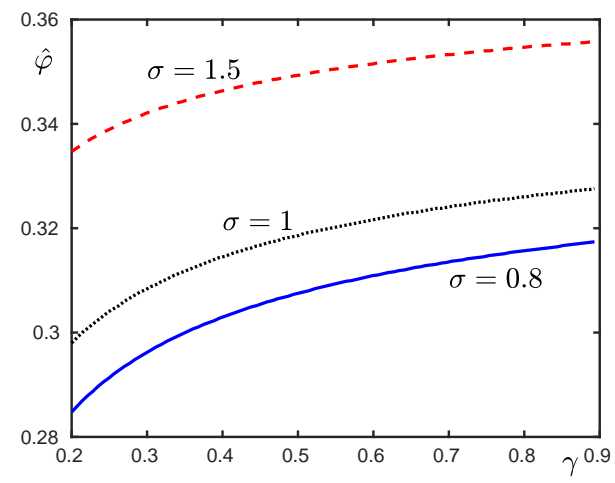

Figure 6: Effect of $\gamma$ on $\hat{\varphi}$.

\section{Concluding remarks}

This paper analyzes a simple AK-endogenous growth model under the following main assumptions: i) a negative externality (e.g. pollution) enters the consumers' utility; ii) time preferences are characterized by a decreasing level of impatience with the timedistance from the present; and iii) current individuals acknowledge that the discount rate of future cohorts will not be as low as anticipated and, hence, try to manipulate their future selves, playing a game against them all (time-consistent individuals). Two main consequences stem from these assumptions. First, the Markov perfect equilibrium in linear strategies for this game, which is a balanced growth path, is characterized by a slower growth when played by current against future central planners than when played by competitive agents, who ignore how investment on production capacity increases pollution. Second, agents with a decaying rate of temporal discount are more long-run oriented than standard individuals with a constant discount rate and the same overall impatience. This can be explained because present-biased individuals strongly discount 
the near future and much less the distant future. Tying these two consequences together we have proved the main finding of our paper: if the pollution externality on utility is sufficiently small, then the strategic interaction between subsequent central planners could lead to a too slow growth rate, so the social welfare of long-run oriented individuals ends up being smaller than in the market economy.

To understand this result it is important to distinguish between what a current individual would like his future selves to do, and the equilibrium of the inter-generational game against these future selves. The equilibrium defines a constant propensity to consume out of wealth, which is a second-best option for the current agent, which is too small in the short run and too large in the long run. In the centralized equilibrium, where the pollution externality is internalized, the constant propensity to consume is high in order to slow down growth (and hence pollution), inducing a positive welfare effect. However, this high propensity to consume in the centralized economy also has a negative welfare effect when the individuals discount at a non-constant rate. Because the current agent would like his far ahead future selves to save more and consume less, the low propensity in the market economy can provide higher long-run welfare than the high propensity in the centralized economy. Because individuals strongly discount the near future, and much less so the far future, they strongly value this higher long-run welfare. We have proven that this second negative effect overcomes the positive one when the pollution externality is small, and hence the market equilibrium provides higher social welfare. Conversely, if the externality is too large, the positive effect prevails and the standard result of a centralized welfare improving equilibrium applies.

We numerically observe that a rise in the EIS widens the range of values for the pollution externality compatible with a market solution providing higher social welfare. Likewise, the more dissimilar from exponential discounting the time preferences are, the more long-run oriented the individuals are. Therefore, we also observe that a discount function more distant from constant discounting (although keeping overall impatience unchanged) also corresponds to a wider range of values for the pollution externality where the market economy welfare dominates the centralized economy.

We have analyzed how the unique balanced growth path, in both the centralized and the market economy, responds to three fundamental aspects: the EIS, the intensity of the pollution externality and the discount function. The value of the EIS has an immediately clear consequence, given the assumption of a non-separable utility function in consumption and pollution. If the EIS is lower than one, as is commonly assumed 
in macroeconomic models, a compensation effect of pollution on consumption fosters economic growth. In contrast, an EIS above one would imply a distaste effect of pollution on consumption, so discouraging growth. Regardless of the existence of a compensation or a distaste effect, a higher EIS always leads to faster growth.

As for the pollution externality, it directly induces growth reductions in the centralized equilibrium. However, for the market equilibrium, if the EIS is lower than one, given the compensation effect, the pollution externality fosters growth. Conversely, when the EIS is greater than one, the distaste effect implies a reduction in economic growth with the pollution externality.

When the instantaneous discount rate decreases, these preferences are more dissimilar from exponential discounting the wider the range of variation for the instantaneous discount rate, or the smoother their decay with the time distance from the present. Considering identical overall impatience, the more dissimilar from exponential discounting, the more strongly the agents discount the nearby future with respect to the far distant future, and the more long-run oriented these individuals are. When the EIS is lower than one, current savings have decreasing future marginal effects on utility. Therefore, the more long-run oriented individuals are, the lower are the aggregate returns to current savings, and a lower incentive to save slows down growth. Conversely, if the EIS is greater than one, the marginal effect of current savings increases with the time distance from the present, and being more long-run oriented leads individuals to enhance savings and growth.

Assuming that agents' degree of impatience decreases with the time distance from the present, our analysis will have policy implications. If pollution stemming from the productive process partially reduces the utility from consumption and this externality is not too large, then the market equilibrium provides a higher welfare than the strategic interaction between subsequent central planners. Under these circumstances no policy actions would be needed. In contrast, these policy actions might be necessary when the externality surpasses a given threshold. This threshold is larger the more dissimilar from exponential discounting the agents are and the greater the EIS. Thus, policy actions would be less necessary in wealthy countries with a well-educated population familiar with financial markets, where according to the empirical evidence the EIS is larger (see, for example, Ben-Gad 2012, Havranek et al. 2015 and Thimme 2017).

The analysis was carried out for a toy AK-model. Under this simple specification we have been able to prove the existence and uniqueness of the centralized and decentralized 
balanced growth path equilibria. Moreover, the reaction of the equilibrium growth rates to changes in the EIS and the pollution externality has also been derived. Finally, the result of a welfare-improving market solution for a sufficiently small pollution externality has also been analytically proved for any plausible EIS. Numerical results could be explored for more complex models. 


\section{A Appendix.}

Lemma 9 When considering an infinite time horizon, the improper integral which collects the present value of the stream of discounted utility $\Omega(g, \sigma, \varphi)$ is convergent if and only if the constant growth rate of the economy satisfies

$$
\eta g+\rho_{\infty}>0
$$

This inequality is satisfied for any $\sigma \leq 1$, as well as if $\sigma>1$ and $g<-\rho_{\infty} / \eta$

\section{Proof.}

From (3), and taking into account that $\rho(j)$ is strictly decreasing towards $\rho_{\infty}>0$, then

$$
\theta(j)=e^{-\int_{0}^{j} \rho(z) d z}<e^{-\rho_{\infty} j}
$$

In consequence, $\Omega(g, \sigma, \varphi)$ is upper bounded:

$$
\Omega(g, \sigma, \varphi)<\int_{0}^{\infty} e^{-\left(\eta g+\rho_{\infty}\right) j} d j .
$$

Therefore, $\eta g+\rho_{\infty}>0$ guarantees the convergence of the integral $\Omega(g, \sigma, \varphi)$.

In contrast, if condition (23) does not hold, then $-\eta g>\rho_{\infty}, \sigma>1$ and $\eta<0$. Therefore, since $\rho(j)$ is strictly decreasing towards $\rho_{\infty}>0$, two possible cases arise:

1. If $\rho(0)>-\eta g$, then there exists a $\breve{j}$ such that $\rho(\check{j})=-\eta g$ and $\rho(z) \in\left(\rho_{\infty},-\eta g\right)$ for all $z>\check{j}$. Thus,

$$
\Omega(g, \sigma, \varphi)=\int_{0}^{\check{j}} e^{-\eta g j} e^{-\int_{0}^{j} \rho(z) d z} d j+\int_{\check{j}}^{\infty} e^{-\eta g j} e^{-\int_{0}^{j} \rho(z) d z} d j .
$$

The second integral is lower bounded:

$$
e^{-\int_{0}^{\check{j}} \rho(z) d z} \int_{\check{j}}^{\infty} e^{-\eta g j} e^{-\int_{\check{j}}^{j} \rho(z) d z} d j>e^{-\int_{0}^{\check{j}} \rho(z) d z} \int_{\check{j}}^{\infty} e^{-\eta g j} e^{\int_{\check{j}}^{j} \eta g d z} d j \equiv e^{-\int_{0}^{\check{j}} \rho(z) d z} \int_{\check{j}}^{\infty} e^{-\eta g \check{j}} d j .
$$

Because this last integral is clearly divergent, this is also true for $\Omega(g, \sigma, \varphi)$.

2. If $\rho(0) \leq-\eta g$, then, on replacing $\check{j}$ by zero in the second integral in (24), and following the same reasoning, it immediately follows that $\Omega(g, \sigma, \varphi)>\int_{0}^{\infty} d j$, and, hence, divergent. 


\section{Proof of Proposition 1.}

Case $\sigma \neq 1$.

Centralized economy

Following Karp (2007), an optimal stationary solution of problem (7)-(8) needs to satisfy the following Bellman equation:

$\int_{t}^{\infty} U\left(C_{t}^{\mathrm{SP}}(\tau),\left(K_{t}^{\mathrm{SP}}\right)^{\lambda}(\tau)\right) \theta(\tau-t) \rho(\tau-t) d \tau=\max _{C_{t}}\left\{U\left(C_{t}, K_{t}^{\lambda}\right)+\left(W^{\mathrm{SP}}\right)^{\prime}\left(K_{t}\right)\left[A K_{t}-C_{t}\right]\right\}$,

where $C_{t}^{\mathrm{SP}}(\tau)$ is the consumption and $\left(K_{t}^{\mathrm{SP}}\right)^{\lambda}(\tau)$ the pollution along the optimal path for the $t$-agent, and $W^{\mathrm{SP}}\left(K_{t}\right)=\int_{t}^{\infty} U\left(C_{t}^{\mathrm{SP}}(\tau),\left(K_{t}^{\mathrm{SP}}\right)^{\lambda}(\tau)\right) \theta(\tau-t) d \tau$ denotes the value function. The argument for variables in the RHS of (25) is the current time $t$ (omitted when no confusion can arise) at which the $t$-agent solves his dynamic problem.

First-order condition for optimality reads:

$$
\frac{\partial U\left(C_{t}, K_{t}^{\lambda}\right)}{\partial C_{t}}=\left(W^{\mathrm{SP}}\right)^{\prime}\left(K_{t}\right)
$$

Along a balanced growth path, from equation (13) the value function can be written as $W^{\mathrm{SP}}\left(K_{t}\right)=W_{0}^{\mathrm{SP}}+W_{1}^{\mathrm{SP}} K_{t}^{-\eta}$, with

$$
W_{0}^{\mathrm{SP}}=\frac{\sigma}{(1-\sigma) \hat{\rho}}, \quad W_{1}^{\mathrm{SP}}=\sigma \frac{\left(\xi^{\mathrm{SP}}\right)^{1-\frac{1}{\sigma}}}{\sigma-1} \Omega\left(g^{\mathrm{SP}}, \sigma, \varphi\right),
$$

where $\xi^{\mathrm{SP}}$ represents the optimal propensity to consume out of capital, to be determined. Plugging $C_{t}^{\mathrm{SP}}(\tau)=\xi^{\mathrm{SP}} K_{t}(\tau)$ into the first-order condition in (26), and taking into account the expression for $W_{1}^{\text {sP }}$, one gets

$$
\xi^{\mathrm{SP}}=\frac{1}{(1-\varphi) \Omega\left(g^{\mathrm{SP}}, \sigma, \varphi\right)}, \quad W_{1}^{\mathrm{SP}}=-\frac{1}{\eta}\left[(1-\varphi) \Omega\left(g^{\mathrm{SP}}, \sigma, \varphi\right)\right]^{\frac{1}{\sigma}} .
$$

Taking into account $\xi^{\mathrm{SP}}$ and the dynamics for the capital stock in (8), the optimal growth rate along the balanced path satisfies

$$
g^{\mathrm{SP}}=A-\xi^{\mathrm{SP}}=A-\frac{1}{(1-\varphi) \Omega\left(g^{\mathrm{SP}}, \sigma, \varphi\right)} .
$$

Integrating $\Omega(g, \sigma, \varphi)$ by parts, one gets

$$
\Omega(g, \sigma, \varphi)=\frac{1}{\eta g}-\frac{1}{\eta g} \int_{0}^{\infty} e^{-\eta g j} \theta(j) \rho(j) d j \Leftrightarrow \Delta(g, \sigma, \varphi)=\frac{1}{\Omega(g, \sigma, \varphi)}-\eta g,
$$

with $\Delta(g, \sigma, \varphi)$ defined in (18). Thus, from (29) the alternative way of characterizing the optimal growth rate in (16) and (18) follows. 
Previous reasoning is valid if and only if $\Omega\left(g^{\mathrm{SP}}, \sigma, \varphi\right)$ converges, which is guarantied under condition (23). Note that condition (19) evaluated at the equilibrium (29) translates into (23). As $\sigma$ tends to the value at which $\eta g^{\mathrm{SP}}+\rho_{\infty}$ vanishes, so $\Omega\left(g^{\mathrm{SP}}, \sigma, \varphi\right)$ tends to infinity. Therefore, from (29) $g^{\mathrm{SP}}$ converges towards $A$. Then, condition (19) grants the convergence. The same reasoning would apply below, in the market economy, taking into account expression (34), and leading to the same value of $\hat{\sigma}$ in (19).

\section{Market economy}

The Bellman equation in the market economy for the optimization problem (10)-(11) reads:

$$
\begin{aligned}
& \int_{t}^{\infty} U\left(c_{t}^{\mathrm{DE}}(\tau),\left(K_{t}^{\mathrm{DE}}\right)^{\lambda}(\tau)\right) \theta(\tau-t) \rho(\tau-t) d \tau= \\
& \max _{c_{t}}\left\{U\left(c_{t}, K_{t}^{\lambda}\right)+\left(W_{k_{t}}^{\mathrm{DE}}\right)^{\prime}\left(k_{t}, K_{t}\right)\left[r k_{t}-c_{t}\right]+\left(W_{K_{t}}^{\mathrm{DE}}\right)^{\prime}\left(k_{t}, K_{t}\right) \dot{K}_{t}\right\},
\end{aligned}
$$

where $c_{t}^{\mathrm{DE}}(\tau)$ is household consumption, $\left(K_{t}^{\mathrm{DE}}\right)^{\lambda}$ the pollution along the optimal path for

the $t$-agent, and $W^{\mathrm{DE}}\left(k_{t}, K_{t}\right)=\int_{t}^{\infty} U\left(c_{t}^{\mathrm{DE}}(\tau),\left(K_{t}^{\mathrm{DE}}\right)^{\lambda}(\tau)\right) \theta(\tau-t) d \tau$, denotes the value function. First-order condition for optimality reads:

$$
\frac{\partial U\left(c_{t}, K_{t}^{\lambda}\right)}{\partial c_{t}}=\frac{\partial W^{\mathrm{DE}}\left(k_{t}, K_{t}\right)}{\partial k_{t}}
$$

Along a balanced growth path, from equation (15) the value function can be written as $W^{\mathrm{DE}}\left(k_{t}, K_{t}\right)=W_{0}^{\mathrm{DE}}+W_{1}^{\mathrm{DE}} k_{t}^{-\eta_{1}} K_{t}^{-\eta_{2}}$, with

$$
W_{0}^{\mathrm{DE}}=W_{0}^{\mathrm{SP}}, \quad W_{1}^{\mathrm{DE}}=\sigma \frac{\left(\xi^{\mathrm{DE}}\right)^{1-\frac{1}{\sigma}}}{\sigma-1} \Omega\left(g^{\mathrm{DE}}, \sigma, \varphi\right),
$$

where $\xi^{\mathrm{DE}}$ represents the optimal propensity to consume out of capital in the market economy.

Plugging $c_{t}^{\mathrm{DE}}(\tau)=\xi^{\mathrm{DE}} k_{t}(\tau)$ into the first-order condition in (32), and taking into account the expression for $W_{1}^{\mathrm{DE}}$, one gets

$$
\xi^{\mathrm{DE}}=\frac{1}{\Omega\left(g^{\mathrm{DE}}, \sigma, \varphi\right)}, \quad W_{1}^{\mathrm{DE}}=\frac{\sigma}{\sigma-1} \Omega\left(g^{\mathrm{DE}}, \sigma, \varphi\right)^{\frac{1}{\sigma}}
$$

Therefore, from (11), the optimal growth rate along the balanced path satisfies

$$
g^{\mathrm{DE}}=r-\xi^{\mathrm{DE}}=A-\frac{1}{\Omega\left(g^{\mathrm{DE}}, \sigma, \varphi\right)} .
$$

Finally, from (30) the expression in (17) follows. 
Case $\sigma=1$.

Following the same methodology as above, one can obtain the value functions for the centralized economy, $W^{1 \mathrm{SP}}\left(K_{t}\right)=W_{0}^{1 \mathrm{SP}}+W_{1}^{1 \mathrm{SP}} \ln K_{t}$ :

$$
W_{0}^{1 \mathrm{SP}}=\frac{g^{1 \mathrm{SP}}(1-\varphi) \bar{J}^{1}+\ln \left(\frac{\hat{\rho}}{1-\varphi}\right)}{\hat{\rho}}, \quad W_{1}^{1 \mathrm{SP}}=\frac{1-\varphi}{\hat{\rho}},
$$

as well as for the market economy, $W^{1 \mathrm{DE}}\left(k_{t}, K_{t}\right)=W_{0}^{1 \mathrm{DE}}+W_{1}^{1 \mathrm{DE}} \ln k_{t}+W_{2}^{1 \mathrm{DE}} \ln K_{t}$ :

$$
W_{0}^{1 \mathrm{DE}}=\frac{g^{1 \mathrm{DE}}(1-\varphi) \bar{J}^{1}+\ln \hat{\rho}}{\hat{\rho}}, \quad W_{1}^{1 \mathrm{DE}}=\frac{1}{\hat{\rho}}, \quad W_{2}^{1 \mathrm{DE}}=-\frac{\varphi}{\hat{\rho}} .
$$

Likewise, the growth rates $g^{1 \mathrm{SP}}$ and $g^{1 \mathrm{DE}}$ in (20) follow.

Lemma 10 Functions $h^{S P}(g)$ and $h^{D E}(g)$ in (16) and (17) are strictly convex for $\sigma \neq 1$ and linear for $\sigma=1$.

Proof. $h^{\mathrm{SP}}(g)$ and $h^{\mathrm{DE}}(g)$ are strictly convex if and only if $\Delta(g, \sigma, \varphi)$ is strictly concave as a function of $g$. To compute the second derivative of $\Delta(g, \sigma, \varphi)$ with respect to $g$, we take into account the first derivative in (38) and the following expressions:

$$
\frac{\partial \bar{J}(g, \sigma, \varphi)}{\partial g}=-\eta \operatorname{Var}(g, \sigma, \varphi), \quad \frac{\partial \Omega(g, \sigma, \varphi)}{\partial g}=-\eta \Omega(g, \sigma, \varphi) \bar{J}(g, \sigma, \varphi),
$$

where $\operatorname{Var}(g, \sigma, \varphi)$ would be the variance when considering $\omega(j)$ as a density function. Thus,

$$
\frac{\partial^{2} \Delta(g, \sigma, \varphi)}{\partial g^{2}}=\frac{\eta^{2}}{\Omega(g, \sigma, \varphi)}\left(\bar{J}^{2}(g, \sigma, \varphi)-\operatorname{Var}(g, \sigma, \varphi)\right) .
$$

To conclude, we need to prove that $\operatorname{Var}(g, \sigma, \varphi)>\bar{J}^{2}(g, \sigma, \varphi)$.

The logarithm of $\omega(j)$ reads:

$$
f(j)=\ln (\omega(j))=B-\eta g j-\int_{0}^{j} \rho(z) d z, \quad \text { where } \quad B=-\ln (\Omega(g, \sigma, \varphi)) .
$$

Because $\dot{\rho}(z)<0$, then $f^{\prime \prime}(j)>0$ and hence $\omega(j)$ is log-convex.

This proof makes use of some of the ideas implemented in Karlin et al. (1961) for log-concave density functions, but applied here for a log-convex function. Following these authors, we define

$$
g(j)=\int_{0}^{\infty} w(t+j) n(t) d t=\int_{j}^{\infty} w(u) n(u-j) d u
$$


where

$$
n(u)= \begin{cases}u^{2} & \text { if } \quad u>0 \\ 0 & \text { if } \quad u \leq 0\end{cases}
$$

Under this specification, provided that $w(j)$ is log-convex, then $g(j)$ is also log-convex (see Boyd and Vandenberghe, 2004, pages 105-106) and, therefore,

$$
g^{\prime \prime}(j) g(j)-\left(g^{\prime}(j)\right)^{2} \geq 0
$$

These derivatives can be computed as

$$
\begin{aligned}
& g^{\prime}(j)=\left(\int_{j}^{\infty} w(u)(u-j)^{2} d u\right)^{\prime}=-2 \int_{j}^{\infty} w(u)(u-j) d u \\
& g^{\prime \prime}(j)=2 \int_{j}^{\infty} w(u) d u .
\end{aligned}
$$

Particularizing for $j=0$, we obtain the first two raw moments: $g(0)=\mu_{2}, g^{\prime}(0)=-2 \mu_{1}$, $g^{\prime \prime}(0)=2$. Thus, inequality $(37) \operatorname{reads} \mu_{2}-2 \mu_{1}^{2} \geq 0$. Or, equivalently $\operatorname{Var}(g, \sigma, \varphi)-$ $\bar{J}^{2}(g, \sigma, \varphi) \geq 0$. Indeed, the inequality is strict unless $f(j)$ refers to the exponential density function, which is both log-concave and log-convex.

Proof of Proposition 3. The derivative of $\omega(j)$ with respect to $g$, reads:

$$
\frac{\partial \omega(j)}{\partial g}=\frac{\theta(j) e^{-\eta g j}}{\Omega(g, \sigma, \varphi)} \eta[\bar{J}(g, \sigma, \varphi)-j], \quad \text { with } \quad \bar{J}(g, \sigma, \varphi)=\int_{0}^{\infty} j \omega(j) d j .
$$

From this expression it is straightforward to compute the derivative of $\Delta(g, \sigma, \varphi)$ with respect to $g$ :

$$
\frac{\partial \Delta(g, \sigma, \varphi)}{\partial g}=-\eta\left(1-\frac{\bar{J}(g, \sigma, \varphi)}{\Omega(g, \sigma, \varphi)}\right)=-\eta \operatorname{Cov}(g, \sigma, \varphi) \gtrless 0 \text { if } \sigma \lessgtr 1 .
$$

Note that the term in round brackets above can be proved to be the covariance between $j$ and $\rho(j)$ (denoted as $\operatorname{Cov}(g, \sigma, \varphi)$ ), which takes a negative value (see, Cabo et al. 2017).

From (38) and the definitions in (16) and (17) one can conclude that $h^{\mathrm{SP}}(g), h^{\mathrm{DE}}(g)$ decrease (increase) with the growth rate of the economy if $\sigma$ is lower (greater) than one. Moreover, from (18) it follows that

$$
\Delta(0, \sigma, \varphi)=\int_{0}^{\infty} \rho(j) \frac{\theta(j)}{\int_{0}^{\infty} \theta(i) d i}=\hat{\rho} \int_{0}^{\infty}-\dot{\theta}(j) d j=\hat{\rho} .
$$

Thus, if (6) is satisfied,

$$
h^{\mathrm{SP}}(0)=\left(A-\frac{\hat{\rho}}{1-\varphi}\right) \sigma>0, \quad h^{\mathrm{DE}}(0)=\frac{A-\hat{\rho}}{1-\varphi+\sigma \varphi} \sigma>0 .
$$


For $\sigma \leq 1,\left(h^{\mathrm{SP}}\right)^{\prime}(g),\left(h^{\mathrm{DE}}\right)^{\prime}(g) \leq 0$ for all $g \in[0, A]$, therefore, an equilibrium, $g^{\mathrm{SP}}$, $g^{\mathrm{DE}}$, always exists and is unique.

For $\sigma>1,\left(h^{\mathrm{SP}}\right)^{\prime}(g),\left(h^{\mathrm{DE}}\right)^{\prime}(g)>0$ for all $g \in[0, A]$, and existence requires $h^{\mathrm{SP}}(A), h^{\mathrm{DE}}(A)<$ $A$. Under condition (6) both inequalities are satisfied if and only if

$$
\sigma<1+\frac{\Delta(A, \sigma, \varphi)}{A(1-\varphi)-\Delta(A, \sigma, \varphi)} .
$$

Note that the denominator in this expression is positive under condition (6) because $\Delta(g, \sigma, \varphi)$ decreases with $g$ when the EIS is larger than one (see (38)). From the definition in (18) it follows that $\Delta(g, \sigma, \varphi) \geq \rho_{\infty}$ for all $g \in[0, A]$. Moreover, function $x /(A(1-$ $\varphi)-x$ ) increases with $x$ and, therefore,

$$
\sigma<\hat{\sigma}<1+\frac{\Delta(A, \sigma, \varphi)}{A(1-\varphi)-\Delta(A, \sigma, \varphi)}
$$

Because curves $h^{\mathrm{SP}}(g), h^{\mathrm{DE}}(g)$ have no inflection points (see Lemma 10), a balanced growth path equilibrium exists in the centralized and in the decentralized economy, as shown in Figure $7 .^{14}$
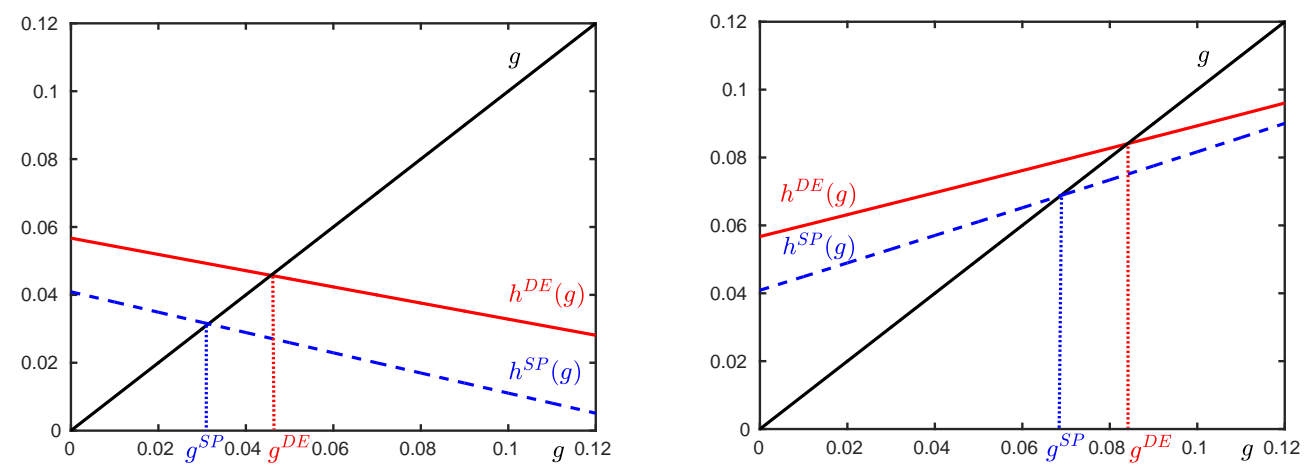

Figure 7: Equilibrium growth rates, $g^{\mathrm{SP}}$ and $g^{\mathrm{DE}}$, for $\sigma<1$ (left) $\sigma>1$ (right).

Moreover, the equilibrium growth rate is larger in the market economy. It can be proved that $h^{\mathrm{DE}}(g)>h^{\mathrm{SP}}(g)$ for all $g \in[0, A]$ if and only if

$$
\sigma<1+\frac{\Delta(g, \sigma, \varphi)}{A(1-\varphi)-\Delta(g, \sigma, \varphi)},
$$

which holds true under the same reasoning.

Lemma 10 proves that $h^{\mathrm{SP}}(g)$ and $h^{\mathrm{DE}}(g)$ are convex functions of $g$. Therefore, since $h^{\mathrm{SP}}(0), h^{\mathrm{DE}}(0)>0$ and $h^{\mathrm{SP}}(A), h^{\mathrm{DE}}(A)<A$, the equilibrium in each scenario is unique.

\footnotetext{
${ }^{14}$ In order to be able to plot these graphs we have considered the particular discount function in (21), which will be commented on in Section 3 .
} 
From this uniqueness it follows that $\left(h^{\mathrm{SP}}\right)^{\prime}\left(g^{\mathrm{SP}}\right),\left(h^{\mathrm{DE}}\right)^{\prime}\left(g^{\mathrm{DE}}\right)<1$. In consequence, since $h^{\mathrm{DE}}(g)>h^{\mathrm{SP}}(g)$ for all $g \in[0, A]$, then $g^{\mathrm{DE}}>g^{\mathrm{SP}}$ as shown in Figure 7 .

\section{Proof of Proposition 4.}

The proof is straightforward from (38).

\section{Proof of Proposition 5.}

From (8) and (16), the dynamic system in the central planner economy reads:

$$
\begin{aligned}
g_{K}=\frac{\dot{K}_{t}}{K_{t}} & =A-\frac{C_{t}}{K_{t}}=A-\hat{c}_{t}, \\
g_{C}=\frac{\dot{C}_{t}}{C_{t}} & =\frac{\sigma}{1-\varphi}\left((1-\varphi) A-\Delta\left(g_{C}, \sigma, \varphi\right)\right) .
\end{aligned}
$$

Along a balanced growth path $g_{K}=g_{C}$ iff $g_{\hat{c}_{t}}=0$, where

$$
g_{\hat{c}}=\frac{\dot{\hat{c}}_{t}}{\hat{c}_{t}}=(\sigma-1) A-\sigma \frac{\Delta\left(A-\hat{c}_{t}, \sigma, \varphi\right)}{1-\varphi}+\hat{c}_{t} .
$$

The steady state for this equation is

$$
\hat{c}_{t}^{\mathrm{SP}}=\left(\frac{C_{t}}{K_{t}}\right)^{\mathrm{SP}}=\sigma \frac{\Delta\left(g^{\mathrm{SP}}, \sigma, \varphi\right)}{1-\varphi}-(\sigma-1) A, \quad \text { with } g^{\mathrm{SP}}=A-\hat{c}_{t}^{\mathrm{SP}} .
$$

Note that

$$
\left.\frac{d \dot{\hat{c}_{t}}}{d \hat{c}_{t}}\right|_{\hat{c}_{t}=\hat{c}_{t}^{\mathrm{SP}}}=\hat{c}_{t}^{\mathrm{SP}}\left(\frac{\sigma}{1-\varphi} \frac{\partial \Delta}{\partial g}\left(g^{\mathrm{SP}}, \sigma, \eta\right)+1\right)=\hat{c}_{t}^{\mathrm{SP}}\left(1-h^{\prime}\left(g^{\mathrm{SP}}\right)\right)>0 .
$$

The last inequality follows because $h^{\prime}\left(g^{\mathrm{SP}}\right)<1$, as proved in the proof of Proposition 3. This proves that the equilibrium $\hat{c}^{\mathrm{SP}}$ is unstable and hence the transitional dynamics to the equilibrium does not arise. Following the same steps one can also prove the instability in the market equilibrium.

Proof of Proposition 6. The direct effect of $\varphi$ on $h^{\mathrm{SP}}(g)$ and $h^{\mathrm{DE}}(g)$ can be computed as

$$
\begin{aligned}
& \frac{\partial h^{\mathrm{SP}}(g)}{\partial \varphi}=-\sigma \frac{\Delta(g, \sigma, \varphi)}{(1-\varphi)^{2}}<0, \\
& \frac{\partial h^{\mathrm{DE}}(g)}{\partial \varphi}=\sigma \frac{(A-\Delta(g, \sigma, \varphi))(1-\sigma)}{(1-\varphi(1-\sigma))^{2}} \gtrless 0 \text { if } \sigma \lessgtr 1 .
\end{aligned}
$$

To compute the indirect effect of $\varphi$, one needs to first compute its effect on $\Delta(g, \sigma, \varphi)$. From (18) it follows that

$$
\frac{\partial \Delta(g, \sigma, \varphi)}{\partial \varphi}=g \frac{1-\sigma}{\sigma} \operatorname{Cov}(g, \sigma, \varphi) \lessgtr 0 \text { if } \sigma \lessgtr 1 .
$$


In consequence, the indirect effect of $\varphi$ through $\Delta(g, \sigma, \varphi)$ reads:

$$
\begin{aligned}
& \frac{\partial h^{\mathrm{SP}}(g)}{\partial \Delta} \frac{\partial \Delta(g, \sigma, \varphi)}{\partial \varphi}=-g \frac{1-\sigma}{1-\varphi} \operatorname{Cov}(g, \sigma, \varphi) \gtrless 0 \text { if } \sigma \lessgtr 1, \\
& \frac{\partial h^{\mathrm{DE}}(g)}{\partial \Delta} \frac{\partial \Delta(g, \sigma, \varphi)}{\partial \varphi}=-g \frac{1-\sigma}{1-\varphi(1-\sigma)} \operatorname{Cov}(g, \sigma, \varphi) \gtrless 0 \text { if } \sigma \lessgtr 1 .
\end{aligned}
$$

In the market economy, adding together direct and indirect effects, curve $h^{\mathrm{DE}}(g)$ shifts up with $\varphi$ when $\sigma<1$, and shifts down when $\sigma>1$. Therefore $g^{\mathrm{DE}}$ grows (resp. decreases) with $\varphi$ when $\sigma<1$ (resp. $\sigma>1$ ). From the expressions in (40) and (42) it is straightforward to conclude that $\varphi$ does not affect $g^{\mathrm{DE}}$ when $\sigma=1$.

In the centralized economy, the direct effect is always negative. Moreover, the indirect effect is also negative for $\sigma>1$, and then the pollution externality undoubtedly shifts $h(g)$ downward. Conversely, when $\sigma<1$ the indirect effect is positive and the total effect can be computed adding together direct and indirect effects, and taking into account (30):

$$
\frac{d h^{\mathrm{SP}}(g)}{d \varphi}=\frac{(1-\sigma) g}{(1-\varphi) \Omega(g, \sigma, \varphi)}\left(\bar{J}(g, \sigma, \varphi)-\frac{1}{\eta g}\right) .
$$

While $\bar{J}(g, \sigma, \varphi)$ is the mean value for the $\omega(j)$ density function in $(18)$, the term $1 /(\eta g)$ can be regarded as the mean value for an exponential density function, $\eta g e^{-\eta g j}$. At $j=0, \omega(0)=1 / \Omega(g, \sigma, \varphi)$ is greater than $\eta g$ (otherwise $\Delta(g, \sigma, \varphi)$ in (30) would be negative). Moreover, since

$$
\frac{d\left(\frac{\theta(j)}{\Omega(g, \sigma, \varphi)}-\eta g\right)}{d j}<0,
$$

the two density functions cross only once, the tail in the exponential is thicker, and therefore,

$$
\bar{J}(g, \sigma, \varphi)<\frac{1}{\eta g} .
$$

The total derivative is negative, and hence $h^{\mathrm{SP}}(g)$ shifts downward also for $\sigma<1$. In consequence $g^{\mathrm{SP}}$ decreases with $\varphi$ regardless of the value of $\sigma$.

\section{Proof of Proposition 7.}

Case $\sigma \neq 1$.

The value functions in the centralized and the market scenarios read:

$$
W^{\mathrm{SP}}\left(K_{t}\right)=W_{0}^{\mathrm{SP}}+W_{1}^{\mathrm{SP}} K_{t}^{-\eta}, \quad W^{\mathrm{DE}}\left(k_{t}, K_{t}\right)=W_{0}^{\mathrm{DE}}+W_{1}^{\mathrm{DE}} k_{t}^{-\eta_{1}} K_{t}^{-\eta_{2}},
$$

where $\eta_{1}+\eta_{2}=\eta$. Taking into account that $k_{t}=K_{t}, W_{0}^{\mathrm{SP}}=W_{0}^{\mathrm{DE}}$ and the expressions of $W_{1}^{\mathrm{SP}}$ and $W_{1}^{\mathrm{DE}}$ in (28) and (33), the difference follows:

$$
W^{\mathrm{DE}}\left(K_{t}, K_{t}\right)-W^{\mathrm{SP}}\left(K_{t}\right)=K_{t}^{-\eta} H(\varphi),
$$


where

$$
H(\varphi)=-\frac{\sigma}{1-\sigma}\left[\Omega\left(g^{\mathrm{DE}}, \sigma, \varphi\right)^{\frac{1}{\sigma}}-(1-\varphi)^{\frac{1-\sigma}{\sigma}} \Omega\left(g^{\mathrm{SP}}, \sigma, \varphi\right)^{\frac{1}{\sigma}}\right] .
$$

From (16) and (17) when $\varphi=0$ it follows that $g^{\mathrm{SP}}=g^{\mathrm{DE}}$ and, hence, $H(0)=0$. In what follows we prove that $H^{\prime}(0)>0$, which implies the existence of a non-empty interval for $\varphi$ within which $H(\varphi)>0$, implying $W^{\mathrm{DE}}\left(K_{t}, K_{t}\right)>W^{\mathrm{SP}}\left(K_{t}\right)$.

To simplify the notation, henceforth we skip the arguments in functions $\Omega, \Delta$ and $\bar{J}$. Thus, we denote:

$$
\begin{array}{lll}
\Omega^{\mathrm{SP}}=\Omega\left(g^{\mathrm{SP}}, \sigma, \varphi\right), & \Delta^{\mathrm{SP}}=\Delta\left(g^{\mathrm{SP}}, \sigma, \varphi\right), & \bar{J}^{\mathrm{SP}}=\bar{J}\left(g^{\mathrm{SP}}, \sigma, \varphi\right), \\
\Omega^{\mathrm{DE}}=\Omega\left(g^{\mathrm{DE}}, \sigma, \varphi\right), & \Delta^{\mathrm{DE}}=\Delta\left(g^{\mathrm{DE}}, \sigma, \varphi\right), & \bar{J}^{\mathrm{DE}}=\tilde{\bar{J}}\left(g^{\mathrm{SP}}, \sigma, \varphi\right) .
\end{array}
$$

The derivative, $H^{\prime}(\varphi)$ reads:

$H^{\prime}(\varphi)=-\frac{1}{1-\sigma}\left[\left(\Omega^{\mathrm{DE}}\right)^{\frac{1-\sigma}{\sigma}} \frac{d \Omega^{\mathrm{DE}}}{d \varphi}+(1-\sigma)(1-\varphi)^{\frac{1-2 \sigma}{\sigma}}\left(\Omega^{\mathrm{SP}}\right)^{\frac{1}{\sigma}}-(1-\varphi)^{\frac{1-\sigma}{\sigma}}\left(\Omega^{\mathrm{SP}}\right)^{\frac{1-\sigma}{\sigma}} \frac{d \Omega^{\mathrm{SP}}}{d \varphi}\right]$.

Taking into account that (16) and (17) implicitly define $g^{\mathrm{SP}}$ and $g^{\mathrm{DE}}$ as functions of $\varphi$, the derivatives above can be written as

$$
\frac{d \Omega^{\mathrm{SP}}}{d \varphi}=\frac{\partial \Omega^{\mathrm{SP}}}{\partial g} \frac{d g^{\mathrm{SP}}}{d \varphi}+\frac{\partial \Omega^{\mathrm{SP}}}{\partial \varphi}, \quad \frac{d \Omega^{\mathrm{DE}}}{d \varphi}=\frac{\partial \Omega^{\mathrm{DE}}}{\partial g} \frac{d \tilde{g}^{*}}{d \varphi}+\frac{\partial \Omega^{\mathrm{DE}}}{\partial \varphi} .
$$

The partial derivatives of $\Omega^{\mathrm{DE}}$ and $\Omega^{\mathrm{SP}}$ w.r.t. $\varphi$ can be computed taking into account (30), (38) and (41):

$$
\begin{array}{ll}
\frac{\partial \Omega^{\mathrm{SP}}}{\partial g}=-\eta \Omega^{\mathrm{SP}} \bar{J}^{\mathrm{SP}}, & \frac{\partial \Omega^{\mathrm{SP}}}{\partial \varphi}=\frac{1-\sigma}{\sigma} g^{\mathrm{SP}} \Omega^{\mathrm{SP}} \bar{J}^{\mathrm{SP}} \\
\frac{\partial \Omega^{\mathrm{DE}}}{\partial g}=-\eta \Omega^{\mathrm{DE}} \bar{J}^{\mathrm{DE}}, & \frac{\partial \Omega^{\mathrm{DE}}}{\partial \varphi}=\frac{1-\sigma}{\sigma} g^{\mathrm{DE}} \Omega^{\mathrm{DE}} \bar{J}^{\mathrm{DE}}
\end{array}
$$

Implicit differentiation in (16) and (17) leads to

$$
\frac{d g^{\mathrm{SP}}}{d \varphi}=\frac{\frac{\partial h^{\mathrm{SP}}\left(g^{\mathrm{SP}}\right)}{\partial \varphi}+\frac{\partial h^{\mathrm{SP}}\left(g^{\mathrm{SP}}\right)}{\partial \Delta} \frac{\partial \Delta^{\mathrm{SP}}}{\partial \varphi}}{1-\left(h^{\mathrm{SP}}\right)^{\prime}\left(g^{\mathrm{SP}}\right)}, \quad \frac{d g^{\mathrm{DE}}}{d \varphi}=\frac{\frac{\partial h^{\mathrm{DE}}\left(g^{\mathrm{DE}}\right)}{\partial \varphi}+\frac{\partial h^{\mathrm{DE}}\left(g^{\mathrm{DE}}\right)}{\partial \Delta} \frac{\partial \Delta^{\mathrm{DE}}}{\partial \varphi}}{1-\left(h^{\mathrm{DE}}\right)^{\prime}\left(g^{\mathrm{DE}}\right)} .
$$

The total effect of $\varphi$ on $h^{\mathrm{SP}}\left(g^{\mathrm{SP}}\right)$ is computed in (43). Likewise, taking into account (40), (42), (30) and (34), the total effect of $\varphi$ on $h^{\mathrm{DE}}\left(g^{\mathrm{DE}}\right)$, after some computation reads:

$$
\frac{d h^{\mathrm{DE}}\left(g^{\mathrm{DE}}\right)}{d \varphi}=\sigma \frac{1-\sigma}{\Omega^{\mathrm{DE}}(1-\varphi(1-\sigma))^{2}} g^{\mathrm{DE}} \bar{J}^{\mathrm{DE}}(1+\eta) .
$$

Moreover, from (38) it follows that

$$
\begin{aligned}
& \left(h^{\mathrm{SP}}\right)^{\prime}\left(g^{\mathrm{SP}}\right)=-\frac{\sigma}{1-\varphi} \frac{\partial \Delta^{\mathrm{SP}}}{\partial g}=(1-\sigma) \operatorname{Cov}\left(g^{\mathrm{SP}}, \sigma, \varphi\right), \\
& \left(h^{\mathrm{DE}}\right)^{\prime}\left(g^{\mathrm{DE}}\right)=-\frac{\sigma}{1-\varphi(1-\sigma)} \frac{\partial \Delta^{\mathrm{DE}}}{\partial g}=\frac{\sigma \eta}{1-\varphi(1-\sigma)} \operatorname{Cov}\left(g^{\mathrm{DE}}, \sigma, \varphi\right) .
\end{aligned}
$$


Plugging all these expressions into (44) and evaluating at $\varphi=0$, when it holds true that $g^{\mathrm{SP}}=g^{\mathrm{DE}}, \Omega^{\mathrm{SP}}=\Omega^{\mathrm{DE}}, \bar{J}^{\mathrm{SP}}=\bar{J}^{\mathrm{DE}}$ and $\Delta^{\mathrm{SP}}=\Delta^{\mathrm{DE}}$, then

$$
H^{\prime}(0)=\left(\Omega^{\mathrm{SP}}\right)^{\frac{1}{\sigma}}\left\{\left[\frac{d g^{\mathrm{DE}}}{d \varphi}-\frac{d g^{\mathrm{SP}}}{d \varphi}\right] \frac{\bar{J}^{\mathrm{SP}}}{\sigma}-1\right\},
$$

where, taking into account that $\operatorname{Cov}(g, \sigma, \varphi)=1-\bar{J}^{\mathrm{SP}} / \Omega^{\mathrm{SP}}$,

$$
\frac{d g^{\mathrm{DE}}}{d \varphi}-\frac{d g^{\mathrm{SP}}}{d \varphi}=\frac{\sigma}{\sigma \Omega^{\mathrm{SP}}+(1-\sigma) \bar{J}^{\mathrm{SP}}}
$$

and, therefore,

$$
H^{\prime}(0)=\left(\Omega^{\mathrm{SP}}\right)^{\frac{1}{\sigma}} \frac{\sigma\left(\bar{J}^{\mathrm{SP}}-\Omega^{\mathrm{SP}}\right)}{\sigma \Omega^{\mathrm{SP}}+(1-\sigma) \bar{J}^{\mathrm{SP}}}=\left(\Omega^{\mathrm{SP}}\right)^{\frac{1}{\sigma}} \frac{-\operatorname{Cov}\left(g^{\mathrm{SP}}, \sigma, 0\right) \sigma}{1-(1-\sigma) \operatorname{Cov}\left(g^{\mathrm{SP}}, \sigma, 0\right)} .
$$

Finally, given that $h^{\mathrm{SP}}(0)>0$ and $h^{\mathrm{SP}}(A)<A$, then the uniqueness of the equilibrium guarantees that $\left(h^{\mathrm{SP}}\right)^{\prime}\left(g^{\mathrm{SP}}\right)<1$. From (45) this inequality is equivalent to $\sigma \Omega^{\mathrm{SP}}+(1-$ $\sigma) \bar{J}^{\mathrm{SP}}>0$ i.e. $1-(1-\sigma) \operatorname{Cov}\left(g^{\mathrm{SP}}, \sigma, 0\right)>0$. Therefore, $H^{\prime}(0)>0$, which concludes the proof.

Case $\sigma=1$.

Following similar reasoning, the gap between the value functions under the market and the centralized solutions can be obtained from (20), (35) and (36):

$$
W^{1 \mathrm{DE}}\left(K_{t}, K_{t}\right)-W^{1 \mathrm{SP}}\left(K_{t}\right)=H^{1}(\varphi)=\varphi \bar{J}^{1}+\frac{\ln (1-\varphi)}{\hat{\rho}} .
$$

This function satisfies $H^{1}(0)=0$ and

$$
\left(H^{1}\right)^{\prime}(0)=\bar{J}^{1}-\frac{1}{\hat{\rho}}=\bar{J}^{1}-\Omega^{\mathrm{SP}}\left(g^{\mathrm{SP} 1}, 1,0\right)=-\Omega^{\mathrm{SP}}\left(g^{\mathrm{SP} 1}, 1, \varphi\right) \operatorname{Cov}\left(g^{\mathrm{SP} 1}, 1,0\right)>0,
$$

which concludes the proof.

\section{Proof of Proposition 8.}

The direct effect of $\sigma$ on $h^{\mathrm{SP}}(g)$ and $h^{\mathrm{DE}}(g)$ can be computed as

$$
\begin{aligned}
& \frac{\partial h^{\mathrm{SP}}(g)}{\partial \sigma}=A-\frac{\Delta(g, \sigma, \varphi)}{1-\varphi}>0, \\
& \frac{\partial h^{\mathrm{DE}}(g)}{\partial \sigma}=\frac{(A-\Delta(g, \sigma, \varphi))(1-\varphi)}{(1-\varphi(1-\sigma))^{2}}>0 .
\end{aligned}
$$

To compute the indirect effect of $\sigma$, one needs to first compute its effect on $\Delta(g, \sigma, \varphi)$. From (18) and (38) it follows that

$$
\frac{\partial \Delta(g, \sigma, \varphi)}{\partial \sigma}=g \frac{1-\varphi}{\sigma^{2}} \operatorname{Cov}(g, \sigma, \varphi)<0 .
$$


In consequence, the indirect effect of $\sigma$ through $\Delta(g, \sigma, \varphi)$ would also be positive:

$$
\begin{aligned}
& \frac{\partial h^{\mathrm{SP}}(g)}{\partial \Delta} \frac{\partial \Delta(g, \sigma, \varphi)}{\partial \sigma}=-g \frac{1}{\sigma} \operatorname{Cov}(g, \sigma, \varphi)>0, \\
& \frac{\partial h^{\mathrm{DE}}(g)}{\partial \Delta} \frac{\partial \Delta(g, \sigma, \varphi)}{\partial \sigma}=-g \frac{1-\varphi}{\sigma(1-\varphi(1-\sigma))} \operatorname{Cov}(g, \sigma, \varphi)>0 .
\end{aligned}
$$

Adding the direct and indirect effects, curves $h^{\mathrm{SP}}(g)$ and $h^{\mathrm{DE}}(g)$ shift upward with $\sigma$. For $\sigma \leq 1,\left(h^{\mathrm{SP}}\right)^{\prime}(g),\left(h^{\mathrm{DE}}\right)^{\prime}(g) \leq 0$ as shown in Figure 7 (left). Hence higher $\sigma$ undoubtedly leads to higher equilibrium growth rates. For $\sigma>1,\left(h^{\mathrm{SP}}\right)^{\prime}(g),\left(h^{\mathrm{DE}}\right)^{\prime}(g)>0$ and $h^{\mathrm{SP}}(0), h^{\mathrm{DE}}(0)$ in $(39)$ are strictly positive, as depicted in Figure 7 (right). Uniqueness implies $\left(h^{\mathrm{SP}}\right)^{\prime}\left(g^{\mathrm{SP}}\right),\left(h^{\mathrm{DE}}\right)^{\prime}\left(g^{\mathrm{DE}}\right)<1$ and hence also ensures a positive effect of $\sigma$ on the equilibrium growth rates.

\section{References}

[2] Barro, R.J. (1999). Ramsey meets Laibson in the Neoclassical growth model. Quarterly Journal of Economics 114, 1125-1152.

[3] Ben-Gad, M (2012). The two sector endogenous growth model: An atlas. Journal of Macroeconomics 34, 706-722

[4] Boyd S. and Vandenberghe L. (2004). Convex Optimization, Cambridge University Press, Cambridge, UK.

[5] Cabo, F., Martín-Herrán, G. and Martínez-García, M.P. (2015). Non-constant discounting and Ak-type growth models. Economics Letters 131, 54-58.

[6] Cabo, F., Martín-Herrán, G. and Martínez-García, M.P. (2016). Unbounded growth in the Neoclassical growth model with non-constant discounting. Mathematical Social Sciences 84, 93-104.

[7] Cabo, F., Martín-Herrán, G. and Martínez-García, M.P. (2017). Non-constant Discounting, Social Welfare and Endogenous Growth with Pollution Externalities. Les Cahiers du GERAD G-2017-85.

[8] Farzin, Y.H. and Wendner, R. (2014). The time path of the saving rate: hyperbolic discounting and short-term planning. MPRA Paper No. 54614, posted 19. March 2014. 
[9] Gruber, J. (2013). A tax-based estimate of the elasticity of intertemporal substitution. Quarterly Journal of Finance 3(1), June 28.

[10] Hall, R.E. (1988). Intertemporal substitution in consumption. Journal of Political Economy 96, 339-357.

[11] Havranek, T. Horvath, R. Irsova, Z. and Rusnak, M. (2015). Cross-country heterogeneity in intertemporal substitution. Journal of International Economics 96, $100-118$.

[12] Karlin, S., Proschan, F. and Barlow, R.E. (1961). Moment inequalities of Pólya frequency functions. Pacific Journal of Mathematics, 11, 1023-1033.

[13] Karp, L. (2007). Non-constant discounting in continuous time. Journal of Economic Theory, 132, 557-568.

[14] Krusell, P., Kuruşçu, B. and Smith, A. A. Jr. (2002). Equilibrium welfare and government policy with quasi-geometric discounting. Journal of Economic Theory $105,42-72$.

[15] Marín-Solano, J. and Navas, J. (2009). Non-constant discounting in finite horizon: the free terminal time case. Journal of Economic Dynamics \& Control 33, 666-675.

[16] Pollak, R.A. (1968). Consistent Planning. Review of Economic Studies 35, 201-208.

[17] Smulders, S. and Gradus, R. (1996). Pollution abatement and long-term growth. European Journal of Political Economy 12, 505-532.

[18] Strotz, R.H. (1956). Myopia and inconsistency in dynamic utility maximization. Review of Economic Studies 23, 165-80.

[19] Strulik, H. (2015). Hyperbolic discounting and endogenous growth. Economics Letters $126,131-134$.

[20] Thimme, J. (2017). Intertemporal Substitution in Consumption: A Literature Review. Journal of Economic Surveys 31, 226-257.

[21] Tsoukis, C., Tournemaine, F. and Gillman, M. (2017). Hybrid exponentialhyperbolic discounting and growth without commitment. The Manchester School, $85(2), 45-74$. 